\title{
Intra-articular corticosteroids are effective in osteoarthritis but there are no clinical predictors of response
}

\author{
Adrian Jones, Michael Doherty
}

\begin{abstract}
Objectives-To show whether intraarticular steroid injections are effective in osteoarthritis; to determine factors that predict response; and to determine whether injection has a beneficial effect on muscle strength.

Methods-Double blind, placebo controlled, crossover study in 59 patients with symptomatic osteoarthritis of the knee.

Outcome measure-Primary outcome measure: change in visual analogue score for pain at three weeks. Predictors of response analysed using logistic regression with a $15 \%$ decrease in pain score at three weeks defining response.

Results-Intra-articular methyl prednisolone acetate produced a significant reduction in visual analogue pain score at three weeks compared to both baseline (median change $\mathbf{- 2 . 0} \mathrm{mm}$, interquartile range -16.25 to 4.0 ) and placebo (median $0.0 \mathrm{~mm}$, interquartile range -9.0 to 6.25 ). No clinical predictors of response could be identified. Muscle strength was not significantly improved in the short term by intra-articular injection.

Conclusions-Intra-articular corticosteroids are effective for short term relief of pain in osteoarthritis but predicting responders is not possible. There may be a place for their more widespread use. Key terms: osteoarthritis; intra-articular steroids; pain relief
\end{abstract}

(Ann Rheum Dis 1996;55:829-832)

The use of intra-articular corticosteroids in osteoarthritis is controversial. Although three studies have shown the efficacy of long acting steroid preparations in knee osteoarthritis ${ }^{1-3}$ there is still some doubt as to how they should be used. It has been suggested that they should be reserved for patients with evidence of synovitis. Two previous studies have attempted to define features that might predict response but could not determine any except clinical effusion. ${ }^{23}$ We felt there was a need to explore this issue further, particularly given current concerns regarding non-steroidal antiinflammatory drugs (NSAID).
Muscle strength is increasingly being recognised as an important determinant of disability in osteoarthritis. ${ }^{4}$ There are data showing that intra-articular steroid injections result in improved muscle strength in knees affected by rheumatoid synovitis. ${ }^{5}$ We wished to determine whether intra-articular steroid injections have a similar beneficial effect on muscle strength in knee osteoarthritis.

\section{Methods}

The study was approved by the local ethics research committee.

PATIENTS

All consecutive patients with hospital-referred, radiographic, symptomatic (painful) knee osteoarthritis were approached for willingness to participate in the study. All fulfilled the American College of Rheumatology criteria for knee osteoarthritis (clinical + radiographic) ${ }^{6}$ and all gave fully informed written consent. Apart from local sepsis or unwillingness to participate there were no other exclusion criteria. All patients continued their normal analgesics and NSAID, and the use of these agents was monitored.

\section{INTERVENTION}

Patients received, in random order, two injections: $40 \mathrm{mg}(1 \mathrm{ml})$ methyl prednisolone acetate; and $1 \mathrm{ml} 0.9 \%$ saline. Each injection was given by a second operator, thus blinding both patient and assessor. The knee was aspirated to apparent dryness before each injection. The injections were separated by an interval of eight weeks.

\section{ASSESSMENTS}

All patients were assessed one week before their participation in the study, then immediately before each injection, and at three and eight weeks after each injection. Assessments at each visit included:

- Pain on a nominated activity, measured using a $100 \mathrm{~mm}$ visual analogue score (VAS)

- Range of movement (degrees)

- Duration of early morning stiffness (minutes)

- Duration of postinactivity stiffness (minutes) 
- Local tenderness (graded 0-3) ${ }^{7}$

- Effusion graded 0-3

- Local heat (present or absent)

- Synovial thickening (present or absent)

- Maximum isometric quadriceps strength measured using a commercial strain gauge with the knee and hip flexed to $90^{\circ}$ (patient sitting, pelvis fixed) ${ }^{8}$

- Hospital anxiety and depression score ${ }^{9}$

- Stanford health assessment questionnaire $(\text { HAQ })^{10}$

All clinical assessments were made by the same blinded observer.

\section{STATISTICAL ANALYSIS}

The primary outcome measure was change in VAS pain score from baseline (time of injection) at three weeks. After first establishing that the change from baseline data approximated to a normal distribution Student's paired $t$ test was employed. This method of analysis was chosen a priori in order to simplify the comparison by removing the confounding effect of time ${ }^{11}$ and to preserve the crossover trial design. Secondary analyses of efficacy were also performed using a two way multiple analysis of variance, either parametric or non-parametric as appropriate for change from baseline at three and eight weeks. As some data was missing due to patient withdrawal, all analyses were performed on a last measures carried forward, intention to treat basis. Preference was assessed using McNemar's $\chi^{2}$ test. To assess the effect of treatment, time, and order, generalised interactive linear modelling using the change from baseline values to preserve matching was used (Minitab).

To assess predictors of response an a priori definition of significant response was employed. This was defined as a $15 \%$ reduction in VAS score at three weeks compared to baseline. We adopted this

Table 1 Baseline characteristics and median change in outcome measures at three and eight weeks following steroid and placebo injection.

\begin{tabular}{|c|c|c|c|c|c|c|}
\hline & \multicolumn{2}{|l|}{ Baseline } & \multicolumn{2}{|c|}{ Change at 3 weeks } & \multicolumn{2}{|c|}{ Change at 8 weeks } \\
\hline & Median & IQ range & Media & IQ range & Media & IQ range \\
\hline \multicolumn{7}{|l|}{ Steroid } \\
\hline VAS pain score & 62.6 & $5.6,68.2$ & -2.0 & $-16.25,4.0$ & 0.0 & $-14.5,8.0$ \\
\hline EMS & 7.0 & $0.75,30.0$ & 0.0 & $-2.25,0.0$ & 0.0 & $-2.25,3.25$ \\
\hline Inactivity stiffness & 1.0 & $0.0,11.25$ & 0.0 & $-1.25,0.0$ & 0.0 & $-1.0,5.0$ \\
\hline Heat & 0.0 & $0.0,0.25$ & 0.0 & $0.0,0.0$ & 0.0 & $0.0,0.0$ \\
\hline Fluid & 1.0 & $0.0,2.0$ & 0.0 & $-1.0,0.0$ & 0.0 & $-1.0,0.0$ \\
\hline Tenderness & 1.0 & $0.0,2.0$ & 0.0 & $0.0,0.0$ & 0.0 & $0.0,0.0$ \\
\hline Range of movement & 97.5 & $90.0,120.0$ & 10.0 & $0.0,41.25$ & 0.0 & $-12.5,10.0$ \\
\hline Anxiety & 6.0 & $3.0,9.0$ & 0.0 & $-1.0,1.0$ & 0.0 & $-1.0,1.0$ \\
\hline Depression & 5.0 & $3.0,7.25$ & 0.0 & $-2.0,0.0$ & 0.0 & $-1.0,1.0$ \\
\hline LHAQ score & 1.5 & $1.0,2.3$ & 0.0 & $-0.3,0.0$ & 0.0 & $-0.3,0.0$ \\
\hline Quads strength & 73.0 & $43.8,102.8$ & 0.0 & $-6.25,6.0$ & 0.0 & $-6.3,11.3$ \\
\hline \multicolumn{7}{|l|}{ Placebo } \\
\hline VAS pain score & 55.5 & $43.75,79.75$ & 0.0 & $-9.0,6.25$ & 0.5 & $-2.0,11.25$ \\
\hline EMS & 5.0 & $1.75,33.75$ & 0.0 & $-4.0,0.0$ & 0.0 & $-2.0,0.0$ \\
\hline Inactivity stiffness & 5.0 & $1.0,30.0$ & 0.0 & $-5.0,0.0$ & 0.0 & $-1.0,0.25$ \\
\hline Heat & 0.0 & $0.0,1.0$ & 0.0 & $-1.0,0.0$ & 0.0 & $0.0,0.0$ \\
\hline Fluid & 1.0 & $0.0,2.0$ & 0.0 & $-1.0,0.0$ & 0.0 & $-1.0,0.0$ \\
\hline Tenderness & 2.0 & $0.0,2.0$ & 0.0 & $0.0,0.0$ & 0.0 & $0.0,0.0$ \\
\hline ROM & 11.0 & $90.0,120.0$ & 10.0 & $0.0,30.0$ & 0.0 & $-10.0,12.5$ \\
\hline Anxiety & 6.0 & $2.0,9.0$ & 0.0 & $-1.0,0.0$ & 0.0 & $-1.0,1.0$ \\
\hline Depression & 4.5 & $3.0,8.0$ & 0.0 & $-1.0,0.25$ & 0.0 & $-1.0,1.0$ \\
\hline LHAQ score & 1.7 & $1.0,2.0$ & 0.0 & $-0.08,0.0$ & 0.0 & $-0.3,0.08$ \\
\hline Quads strength & 67 & $48.5,99$ & 0.0 & $-6.3,3.0$ & -1.0 & $-7.0,2.8$ \\
\hline
\end{tabular}

VAS, visual analogue score; EMS, early morning stiffness; LHAQ lower limb HAQ; IQ interquartile. approach since we felt that using a continuous outcome measure - that is, absolute or relative change in VAS score - had the potential for extreme responders to introduce spuriously high correlations. The data were modelled by stepwise logistic regression using response as the dependent variable (Egret; SERC).

\section{Results}

Sixty patients were studied: 23 males, 37 females, mean age 70.6 years (range 51 to 89 ). One patient failed to enter the study and received no injection, leaving 59 patients available for the analysis. The baseline characteristics of the patients are tabulated (table 1). Twelve patients withdrew prematurely from the study: three developed worsening symptoms (two following steroid, one following placebo); one patient's symptoms resolved (placebo); and eight withdrew for unrelated reasons.

RESPONSE TO INJECTION

Thirty patients favoured the methyl prednisolone injection and 14 the placebo injection (McNemar's $\chi^{2}=14.2$, df $=1, \mathrm{P}<0.001$ ). Using the predetermined criteria, 28 patients responded to the steroid injection, compared to nine responding to the placebo injection ( $P$ $<0.001$, difference in proportions ).

The change in VAS score is shown in the figure. Preliminary analysis showed no effect of order on the change in VAS (table 2) although there was a significant interaction between order and treatment with the mean change (95\% CI) in VAS score in the four groups: placebo first, 1.92 (-3.35 to 7.19); placebo second 3.79 ( -1.74 to 9.32$)$; steroid first -12.91 ( -19.07 to -6.75$)$; steroid second $-7.96(-14.6$ to -1.32$)$. The analyses could thus be performed as outlined in the methods section. A significant reduction in VAS pain score was observed at three weeks compared to both baseline and placebo. The placebo group showed no significant change compared to baseline. The secondary analysis using all four time points showed that there was a significant difference between the groups, and subsequent use of Student's $t$ test for the various possible pairs showed that this difference was due to the reduction of pain VAS at three weeks following steroid injection compared to the placebo group at both time points. All the results are summarised in table 1 .

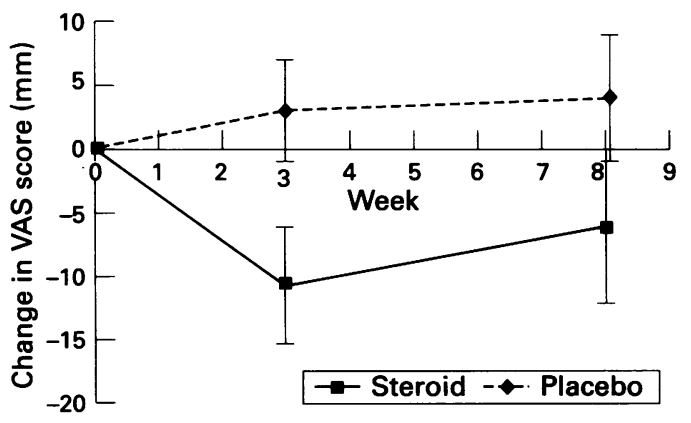

Figure 1 Change in visual analogue score following injection (mean and standard error plotted). 
Table 2 Generalised linear interactive model for change in visual analogue pain score

\begin{tabular}{lllll}
\hline Source & df & Adjusted $M S$ & $F$ statistic & $P$ \\
\hline Order & 1 & 181 & 0.5 & 0.48 \\
Treatment & 1 & 1936.2 & 5.37 & 0.021 \\
(order $\times$ (treatment) & 1 & 7308 & 20.25 & $<0.00001$ \\
Week & 1 & 476 & 1.32 & 0.252 \\
\hline
\end{tabular}

df, degrees of freedom; MS, mean square.

A statistically significant but clinically insignificant reduction in early morning stiffness was also observed. There was no change in the use of NSAID or paracetamol.

PREDICTORS OF PATIENT RESPONSE

The following were entered into a stepwise logistic regression model to determine if there were any simple clinical predictors of patient response: range of movement; fluid; local heat; synovial thickening; tenderness; anxiety score; depression score; HAQ score; lower limb HAQ score; range of movement; quadriceps strength. None was entered in the stepwise logistic regression models as significant predictors of patient's response, although-as can be seen from the crude odds ratios-tenderness was associated with response in univariate analysis (table 3). There were no statistically significant predictors of placebo response either in univariate or multivariate analysis (data not shown).

\section{CHANGE IN MUSCLE STRENGTH FOLLOWING}

INTRA-ARTICULAR STEROID INJECTION

The coefficient of variation of our method of assessing quadriceps strength assessed in healthy volunteers one week apart is $16 \%$. The change in muscle strength following intraarticular steroid injection is tabulated on an intention to treat basis (table 1). Although a significant change in quadriceps strength was seen following steroid injection when analysed on an intention to treat basis, this improvement was not observed when only those with complete data were analysed (data not shown). No change was observed following placebo injection or in the opposite non-injected knee (data not shown).

\section{Discussion}

This study confirms earlier studies that show short lived symptomatic benefit from intra-articular steroid injections. ${ }^{1-3}$ It has thus been argued that such injections should only be given in exceptional circumstances. However, estimates of the side effects of intraarticular steroid injections suggest that these

Table 3 Crude odds ratios for predictors for patient response to steroid injection

\begin{tabular}{lllc}
\hline Baseline characteristic & Odds ratio for response & \multicolumn{2}{l}{$95 \%$ Confidence interval } \\
\hline Age (per year) & 0.98 & 0.92 & 1.04 \\
Gender (male) & 0.46 & 0.15 & 1.40 \\
Local heat (0-1) & 0.85 & 0.28 & 2.58 \\
Local tenderness (0-3) & 1.80 & 1.03 & 1.67 \\
Early morning stiffness (min) & 1.00 & 0.99 & 1.01 \\
Inactivity stiffness (min) & 1.00 & 0.99 & 1.01 \\
Range of movement (degrees) & 0.99 & 0.96 & 1.01 \\
Anxiety score & 1.10 & 0.96 & 1.25 \\
Depression score & 0.94 & 0.80 & 1.11 \\
HAQ score & 1.71 & 0.77 & 3.79 \\
Lower limb HAQ score & 1.47 & 0.73 & 2.96
\end{tabular}

HAQ, health assessment questionnaire. are rare, albeit serious; long term effects on cartilage have not been observed. ${ }^{11}$ Given the importance of adverse reactions to NSAID, perhaps we may be being unduly concerned regarding intra-articular steroid use in symptomatic osteoarthritis.

This study also fails to show any simple clinical predictors of response to steroid injection, although tenderness was a predictor when crude odds ratios are considered. The sample size is small and thus a real effect may have been missed, but it is unlikely that this would be of sufficient predictive value to be of use in directing treatment. This finding is similar to that from one previous study. ${ }^{2}$ Interestingly, aspiration of fluid also did not predict clinical response, in contrast to a previous study. ${ }^{3}$ This is known to be a predictor of intra-articular injection. ${ }^{12}$ Since traditional indices of inflammation (heat, fluid, stiffness) did not predict response to steroids, it may be that local corticosteroids are not acting to relieve pain by reducing synovitis. One might argue that all patients with symptomatic osteoarthritis should be offered a corticosteroid injection to assess response, particularly in those in whom NSAID are contraindicated.

It is also of interest that "inflammation" does not predict response to NSAID. ${ }^{13}$ It may be, however, that current methods of assessing local inflammation in osteoarthritis are inadequate. A significant order effect, as noted in previous studies, was not seen ${ }^{2}$ but there was an interaction between active treatment and order, with more benefit seen if the steroid injection was given first.

Although the intention-to-treat analysis showed an improvement in muscle strength, this must be treated with caution since analysis of those who actually received steroid and were adequately followed up did not confirm this. The failure to show an improvement in quadriceps muscle strength in osteoarthritis following intra-articular injection is of interest since this is not the situation in rheumatoid arthritis. ${ }^{5}$ The nature of the weakness in osteoarthritis is unclear. Muscle wasting is described but the presence of arthrogenic muscle inhibition, a phenomenon that might be readily amenable to alteration by local corticosteroids, is less clear cut. $^{14-16}$

We are grateful to Drs J Ledingham, F Fawthrop, and A Farrell who gave the blinded injections and to Sister S Doherty who helped the organisation of the trial. Since $M D$ is the editor of this journal, impartial review of the paper was arranged by John Axford, acting editor.

1 Friedman DH, Moore ME. The efficacy of intraarticular steroids in osteoarthritis: a double blind study. $\mathcal{F}$ Rheumatol $1980 ; 7: 850-6$

2 Dieppe PA, Sathapatayavongs B, Jones HE, Bacon PA, Ring EFJ. Intra-articular steroids in osteoarthritis. Rheumatol Rehabil 1980;19:212-7.

3 Gaffney K, Ledingham J, Perry JD. Intra-articular triamcinolone hexacetonide in knee osteoarthritis: factors influencing the clinical response. Ann Rheum Dis 1995;54:37981.

4 McAlindon TE, Cooper C, Kirwan JR, Dieppe PA Determinants of disability in osteoarthritis of the knee. Ann Rheum Dis 1993;52:258-62.

5 Geborek P, Månsson B, Wollheim FA, Moritz U. Intraarticular corticosteroid injection into rheumatoid arthritis knees improves extensor muscle strength. Rheumatol Int 1990;9:265-70. 
6 Altman R, Asch E, Bloch D, Bole G, Borenstein D, Brandt $\mathrm{K}$, et al. Development of criteria for the classification and reporting of osteoarthritis: classification of osted

7 Jones A, Hopkinson N, Pattrick M, Berman P, Doherty M. Evaluation of a method for clinically assessing osteoarthritis of the knee. Ann Rheum Dis 1993;51:243-5.

8 Edwards RHT, Young A, Hosking GP, Jones DA. Human skeletal muscle function: description of tests and normal values. Clin Sci 1977;52:283-90.

9 Zigmond AS, Snaith RP. The hospital anxiety and depression scale. Acta Psychiatr Scand 1983;67:361-70.

10 Kirwan JR, Reedback JS. Stanford Health Assessment Questionnaire modified to assess disability in British patients with rheumatoid arthritis. Br f Rheumatol 1986;25:206-9.

11 Sparling M, Malleson P, Wood B, Petty R. Radiographic followup of joints injected with triamcinolone hexacetonide for the management of childhood arthritis. Arthritis Rheum 1990:33:821-6.
12 Jones A, Regan M, Pattrick M, Ledingham J, Doherty M. Importance of placement of intra-articular steroid injections. $B M^{\mathcal{F}}$ 1993;307:1329-30.

13 Bradley JD, Brandt KD, Katz BP, Kalasinski LA, Ryan SI. Treatment of knee osteoarthritis: relationship of clinical features of joint inflammation to the response to a nonsteroidal antiinflammatory drug or pure analgesic. $\mathcal{f}$ Rheumatol 1992;19:1950-4.

14 Jones DW, Jones DA, Newham DJ. Chronic knee effusion and aspiration: the effect on quadriceps inhibition. $\mathrm{Br} \mathcal{F}$ Rheumatol 1987;26:370-4.

15 Hurley MV, Newham DJ. The influence of arthrogenous muscle inhibition on quadriceps rehabilitation of patients with early unilateral osteoarthritic knees. $\mathrm{Br} \mathcal{F}$ Rheumatol 1993;32:127-31.

16 Fahrer H, Rentsch HU, Gerber NJ, Beyeler C, Hess CW, Grunig B. Knee effusion and reflex inhibition of the quadriceps. F Bone foint Surg Br 1988;70B:635-8. 